\title{
Gastroschisis Complicated by Septo-Optic Dysplasia: Two Distinct Anomalies with a Common Origin
}

\author{
Jodi Garvin, DO ${ }^{1}$ Venkatesh Sampath, MRCPCH, MD ${ }^{2}$ Vijender R. Karody, MD² \\ ${ }^{1}$ Department of Pediatrics, Medical College of Wisconsin, Wauwatosa, \\ Children's Hospital of Wisconsin, Milkwaukee, WIsconsin \\ 2 Department of Neonatology, Medical College of Wisconsin, \\ Children's Hospital of Wisconsin, Milwaukee, Wisconsin \\ Address for correspondence Jodi Garvin, DO, Department of \\ Pediatrics, Medical College of Wisconsin, 9000 W. Wisconsin Ave, Mail \\ 756, Milwaukee, WI 53226 (e-mail: jgarvin@mcw.edu).
}

Am J Perinatol Rep 2016;6:e15-e17.

\author{
Abstract \\ Keywords \\ - gastroschisis \\ - septo-optic dysplasia \\ - shock \\ - hypoglycemia
}

Introduction Gastroschisis is considered to be an isolated abdominal wall defect that is infrequently associated with other anomalies.

Case This case describes an infant with gastroschisis who developed refractory shock after an uncomplicated surgery for bowel atresia. He was found to have adrenal insufficiency secondary to septo-optic dysplasia with panhypopituitarism.

Conclusion Gastroschisis and septo-optic dysplasia arise from vascular disruptions, therefore presence in the same infant can be more than just a coincidence. While this is not a common occurrence, our case illustrates the need for a high index of suspicion with an unusual clinical course.
Gastroschisis is an abdominal wall defect usually to the right of the umbilical cord with eventration of abdominal contents without a protective peritoneal covering. This is a common defect with a generally well-defined set of risks and complications; notably premature delivery, prolonged hospitalization, associated bowel atresia, delayed enteral feeds, and total parenteral nutrition (TPN) dependence. ${ }^{1,2}$ We report an infant with gastroschisis who had an atypical postoperative course secondary to panhypopituitarism, a consequence of septo-optic dysplasia (SOD). Both conditions evolve from vascular/thrombotic disruptions thus linking them pathogenically. Although this association is uncommon, prompt recognition and treatment of hormone deficiencies associated with panhypopituitarism may prevent potentially lethal sequelae.

\section{Case}

A Caucasian male baby with gastroschisis identified on the 20 week ultrasound was born to a 22 -year-old primigravida received

April 26, 2015

accepted after revision

July 21, 2015

published online

October 12, 2015 mother. Induction of labor at 34 weeks gestation was elected due to increasing bowel dilation. At the time of birth, he was at the 51st percentile for weight, 33rd percentile for length, and 40th percentile for head circumference on the Fenton growth chart. He was taken to the operating room at 2 hours of life for primary closure of a small $1.5-\mathrm{cm}$ defect without any bowel resection. Postoperatively, he did well. However, by postoperative day 9 , baby had still not passed meconium, and continued to have bilious gastric secretions. The concern for bowel atresia was evident.

Upper gastrointestinal contrast series preformed that day showed midgut malrotation with dilated small bowel loops without progression of contrast. Time was given for stabilization of his respiratory status as well as nutrition. On day of life (DOL) 51 he underwent jejunal resection with $5 \mathrm{~cm}$ of bowel removed as well as Ladd procedure. There were no intraoperative complications; however, postoperatively he developed warm shock that did not improve with volume or inotropes. His cortisol level at the time was 0.5. With the addition of hydrocortisone his hemodynamics improved. By
Copyright $\odot 2016$ by Thieme Medical Publishers, Inc., 333 Seventh Avenue, New York, NY 10001, USA. Tel: +1(212) 584-4662.
License terms

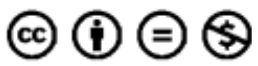

10.1055/s-0035-1563720. ISSN 2157-6998. 
postoperative day 4 he was no longer requiring inotropic support. Empiric antibiotics were stopped after 48 hours when all cultures were negative.

In anticipation of prolonged bowel dysmotility, TPN was initiated on DOL 7 and by DOL 13 he was tolerating 6 hours a day off TPN. On DOL 17 he began having hypoglycemia associated with TPN cycling. Despite decreasing the amount of time off TPN, glucose was still as low as 23. This resolved when TPN was infused continuously. Hypoglycemia occurred again after his second surgery when he was transitioning from TPN to bolus enteral feeds. This corrected when he was put on continuous nasogastric feeds. Due to the recurrence of hypoglycemia, endocrine laboratory work was pursued. This revealed multiple pituitary hormone deficiencies with central hypothyroidism and adrenal insufficiency. With a normal TSH level but inappropriately low in the setting of low T4, hypothyroidism had been missed on newborn screening. An examination performed by endocrine also noted a stretched penile length that was just short of the reference range for his correct gestational age. Baby was started on physiologic hydrocortisone on DOL 83thyroid and growth hormone replacement therapy was started on DOL 85 . By DOL 88 he was tolerating full enteral bolus feeds without any hypoglycemia.

Due to his hormone deficiencies a brain magnetic resonance imaging (MRI) was performed on DOL 102. This showed volume loss of the corpus callosum, hypoplasia of the optic nerves, and optic chiasm. Also, identified was an ectopic posterior pituitary bright spot (-Fig. 1) located outside of its normal location in the sella turcica (-Fig. 2) along with a hypoplastic anterior pituitary gland. These MRI findings were consistent with SOD. Genetics was consulted and they did not recommend genetic testing due to the sporadic nature of SOD. Baby was discharged home on DOL

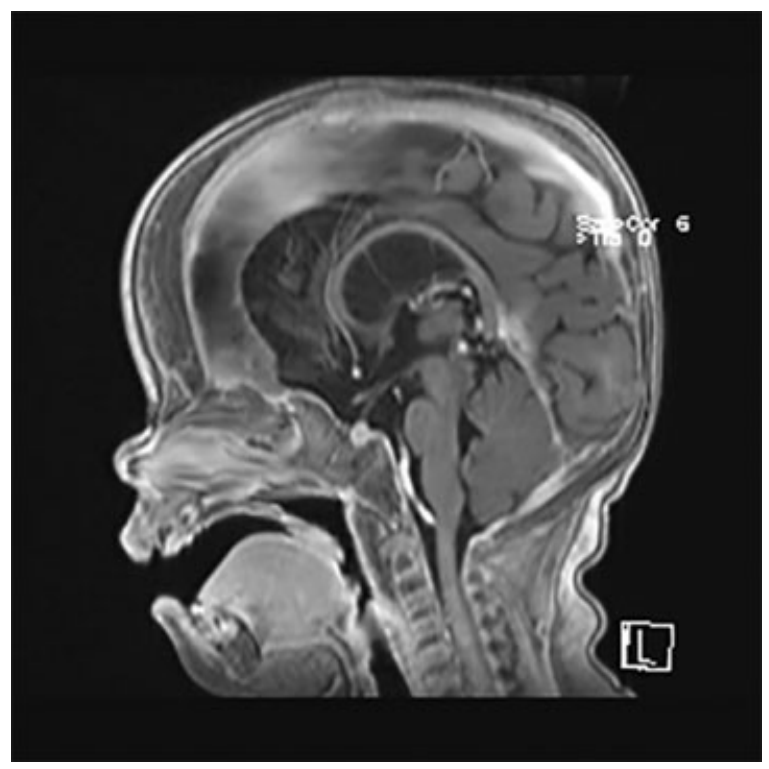

Fig. 1 MRI brain postcontrast T1-weighted image showing the correct location of the posterior pituitary bright spot within the sella turcica. $\mathrm{MRI}$, magnetic resonance imaging.

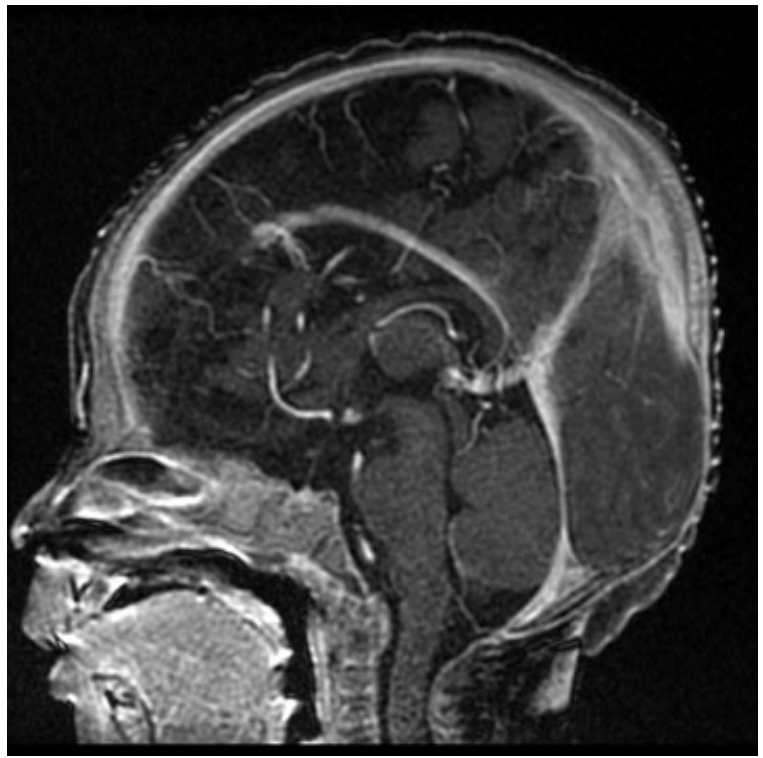

Fig. 2 MRI brain postcontrast T1-weighted image depicts the ectopic location of the posterior pituitary gland. Increased signal can be found at the floor of the third ventricle and not within the sella turcica. MRI, magnetic resonance imaging.

117 taking all feeds by mouth. Follow-up appointments were made with multiple subspecialties.

\section{Discussion}

Gastroschisis effects approximately 4.4 per 10,000 births in the United States and is ranked in the top six most expensive birth defects by the Center for Disease Control.,3 After delivery babies can remain in the neonatal intensive care unit (NICU) for a month or more waiting for bowel function to resume and for feeds to be tolerated. ${ }^{4}$ In general, outcomes are good because of the rare association of gastroschisis with other congenital anomalies. ${ }^{5}$ Our case illustrates an atypical postoperative course due to the hormone deficiencies associated with SOD. Both gastroschisis and SOD have been postulated to be the result of vascular pathology. ${ }^{6,7}$ Therefore, an association of these two entities should be remembered when unusual hospital courses develop.

Although gastroschisis is commonly seen as occurring without other congenital anomalies, studies have been performed that show the contrary. ${ }^{1,5,8}$ One study utilized a database of 348 NICUs in North America. They found that of 4,687 infants born with gastroschisis in a 5-year period, 365 (8\%) had at least one additional congenital anomaly. Within that group, 35 (1\%) specifically had neurological anomalies. ${ }^{1}$ On a more global scale, Mastroiacovo et al retrospectively looked at cumulative data from 24 birth defect registries around the world. They calculated $14 \%$ of 3,322 cases of gastroschisis were nonisolated. Of the nonisolated cases, $4.5 \%$ had an additional neurologic anomaly. ${ }^{8}$ Although these percentages are small, they are not insignificant.

SOD is a clinical diagnosis defined by the presence of two out of three characteristics. The triad consists of optic nerve 
hypoplasia, pituitary hormone abnormalities, and midline brain defects. This is a rare anomaly and is present in 1 out of 10,000 births. ${ }^{9}$ Given the sporadic nature of both SOD and nonisolated gastroschisis, one would think an association between the two is few and far between. However case reports do exist, and multiple studies of gastroschisis outcomes have described the two anomalies occurring together. 4 ,10-15

Historically, the development of gastroschisis has been attributed to a vascular disruption. ${ }^{7,16}$ Likewise, a vascular origin has been hypothesized to be cause of SOD. This is supported by the fact that the three components of SOD arise from different embryonic tissue and develop during different weeks of gestation. More convincingly, the three abnormalities are within the vascular supply of a common artery. ${ }^{6}$ In addition to this similarity, both gastroschisis and SOD occur more frequently in younger mothers. ${ }^{17,18}$ These common factors suggest something the young mother experiences or is exposed to increases the risk of both anomalies developing in their fetus.

Our case is an example of why being aware of the association between gastroschisis and SOD is important. Having a higher index of suspicion of adrenal insufficiency in the setting of refractory shock will lead to faster restoration of perfusion. But more importantly, it would lead to earlier diagnosis and treatment of thyroid and growth hormone deficiencies. Both of these hormones are vital to the growth and neurologic development of neonates.

\section{References}

1 Corey KM, Hornik CP, Laughon MM, McHutchison K, Clark RH, Smith PB. Frequency of anomalies and hospital outcomes in infants with gastroschisis and omphalocele. Early Hum Dev 2014;90(8):421-424

2 Hook-Dufresne DM, Yu X, Bandla V, Imseis E, Moore-Olufemi SD. The economic burden of gastroschisis: costs of a birth defect.J Surg Res 2015;195(1):16-20
3 Kirby RS, Marshall J, Tanner JP, et al; National Birth Defects Prevention Network. Prevalence and correlates of gastroschisis in 15 states, 1995 to 2005. Obstet Gynecol 2013;122(2 Pt 1):275-281

4 Overcash RT, DeUgarte DA, Stephenson ML, et al; University of California Fetal Consortium. Factors associated with gastroschisis outcomes. Obstet Gynecol 2014;124(3):551-557

5 Christison-Lagay ER, Kelleher CM, Langer JC. Neonatal abdominal wall defects. Semin Fetal Neonatal Med 2011;16(3):164-172

6 Lubinsky MS. Hypothesis: septo-optic dysplasia is a vascular disruption sequence. Am J Med Genet 1997;69(3):235-236

7 Hoyme HE, Higginbottom MC, Jones KL. The vascular pathogenesis of gastroschisis: intrauterine interruption of the omphalomesenteric artery. J Pediatr 1981;98(2):228-231

8 Mastroiacovo P, Lisi A, Castilla EE, et al. Gastroschisis and associated defects: an international study. Am J Med Genet A 2007; 143A(7):660-671

9 Fard MA, Wu-Chen WY, Man BL, Miller NR. Septo-optic dysplasia. Pediatr Endocrinol Rev 2010;8(1):18-24

10 van Manen M, Hendson L, Wiley M, Evans M, Taghaddos S, Dinu I. Early childhood outcomes of infants born with gastroschisis. J Pediatr Surg 2013;48(8):1682-1687

11 Clark RH, Walker MW, Gauderer MWL. Factors associated with mortality in neonates with gastroschisis. Eur J Pediatr Surg 2011; 21(1):21-24

12 Bonasoni MP, Reyes J, Cromwell S, Halliday W, Taylor GP, Chiasson DA. Sudden death in the septo-optic dysplasia spectrum. Acad Forensic Pathol 2014;4(3):400-408

13 Suver D, Lee SL, Shekherdimian S, Kim SS. Left-sided gastroschisis: higher incidence of extraintestinal congenital anomalies. Am J Surg 2008;195(5):663-666, discussion 666

14 Jordan MA, Montezuma SR. Septo-optic dysplasia associated with congenital persistent fetal vasculature, retinal detachment, and gastroschisis. Retin Cases Brief Rep 2015;9(2):123-126

15 Kamien B, Zankl A, Gabbett M. Septo-optic dysplasia and associations with amyoplasia and gastroschisis. Birth Defects Res A Clin Mol Teratol 2010;88(6):497-501

16 Lubinsky M. A vascular and thrombotic model of gastroschisis. Am J Med Genet A 2014;164A(4):915-917

17 Feldkamp ML, Carey JC, Sadler TW. Development of gastroschisis: review of hypotheses, a novel hypothesis, and implications for research. Am J Med Genet A 2007;143A(7):639-652

18 Lubinsky MS. Association of prenatal vascular disruptions with decreased maternal age. Am J Med Genet 1997;69(3):237-239 